\title{
Forster, Edgar
}

\section{Feminisierung und Geschlechterdifferenz}

Borst, Eva [Hrsg.]; Casale, Rita [Hrsg.]: Ökonomien der Geschlechter. Opladen ; Farmington Hills : Verlag Barbara Budrich 2007, S. 61-75. - (Jahrbuch Frauen- und Geschlechterforschung in der Erziehungswissenschaft; 3)

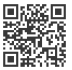

Quellenangabe/ Reference:

Forster, Edgar: Feminisierung und Geschlechterdifferenz - In: Borst, Eva [Hrsg.]; Casale, Rita [Hrsg.]: Ökonomien der Geschlechter. Opladen ; Farmington Hills : Verlag Barbara Budrich 2007, S. 61-75URN: urn:nbn:de:0111-opus-53618 - DOI: 10.25656/01:5361

https://nbn-resolving.arg/um:nbn.de.0111-opus-53618

https://doi.org/10.25656/01:5361

in Kooperation mit / in cooperation with:

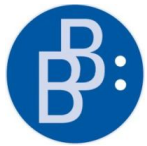

https://mww.budrich.de

\section{Nutzungsbedingungen}

Gewāhnt wird ein nicht exklusives, nicht übertragbares, persōoliches und beschrărnkes Recht auf Nutzung dieses Dokuments. Dieses Dokument ist ausschießlich für den persönlichen. nicht-kommerziellen Gebrauch bestimmt. Die Nutzung stellt keine Ubertragung des Eigentumsrechts an diesem Dokument dar und gill vorbehaltich der folgenden EinschrảnkungenAul sảmticthen Kopien deses Dokuments müssen ale Urheberrechtshirweise und sonstigen Hinweise aut gesetzlichen Schutz beibehallen werden. Sie dürlen dieses Dokument nicht in irgendeiner Weise abändern, nach dürlen Sie dieses Dokument für ölfentiche oder kommerzielle Zwecke verviellältigen, D̈flentlich ausstellen, auffuhren, vertreben oder anderweitig nutzen.

vertreben oder anderweitig mutzen.
Mit der Verwendung dieses Dokuments erkennen Sie die Nutzungsbedingungen an.

\section{Terms of use}

We grant a non-exclusive, non-transferable, individual and limited right to using this document.

This document is solely intended for your personal, non-commercial use. Use of this document does not include amy transfer of property rights and it is conditional to the following limitations: All of the copies of this documents must retain all copyright information and other information regarding legal protection. You are not allowed to alter this document in any way, to copy it for public of commercial purposes, to exhibit the document in public, to perform, distribute or otherwise use the document in public.

By using this particular document, you accept the above-stated conditions of use.

\section{Kontakt / Contact:}

\section{peDOCS}

DIPF | Leibniz-Institut für Bildungsforschung und Bildungsinformation

Informationszentrum (IZ) Bildung

E-Mail: pedocs@dipt.de

Internet: www.pedocs.de

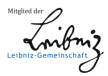


Eva Borst

Rita Casale (Hrsg.)

\section{$3 / 2007$}

Jahrbuch Frauen- und Geschlechterforschung in der Erziehungswissenschaft Ökonomien der Geschlechter 
Ökonomien der Geschlechter 
Jahrbuch der

Frauen- und Geschlechterforschung

in der Erziehungswissenschaft

herausgegeben von

Rita Casale

Barbara Rendtorff

Sabine Andresen

Vera Moser

Annedore Prengel

Beirat

Birgit Althans, Berlin

Eva Borst, Mainz

Eva Breitenbach, Osnabrück

Bettina Dausien, Bielefeld/München

Isabell Diehm, Bielefeld

Hannelore Faulstich-Wieland, Hamburg

Edgar Forster, Salzburg

Edith Glaser, Dortmund

Carola Iller, Heidelberg

Andrea Liesner, Hamburg

Susanne Maurer, Marburg

Inga Pinhard, Frankfurt

Folge 3/2007 
Eva Borst

Rita Casale (Hrsg.)

\section{Ökonomien der Geschlechter}

Verlag Barbara Budrich

Opladen \& Farmington Hills 2007 
Bibliografische Informationen der Deutschen Nationalbibliothek

Die Deutsche Nationalbibliothek verzeichnet diese Publikation in der Deutschen Nationalbibliografie; detaillierte bibliografische Daten sind im Internet über http://dnb.d-nb.de abrufbar.

Gedruckt auf säurefreiem und alterungsbeständigem Papier.

Alle Rechte vorbehalten.

(C) 2007 Verlag Barbara Budrich, Opladen \& Farmington Hills

www.budrich-verlag.de

ISBN 13: 978-3-86649-088-8 / eISBN: 978-3-86649-891-4

Das Werk einschließlich aller seiner Teile ist urheberrechtlich geschützt. Jede Verwertung außerhalb der engen Grenzen des Urheberrechtsgesetzes ist ohne Zustimmung des Verlages unzulässig und strafbar. Das gilt insbesondere für Vervielfältigungen, Übersetzungen, Mikroverfilmungen und die Einspeicherung und Verarbeitung in elektronischen Systemen.

Umschlaggestaltung: disegno visuelle kommunikation, Wuppertal - www.disenjo.de Satz: Beate Glaubitz Redaktion + Satz, Leverkusen

Druck: paper\&tinta, Warschau

Printed in Europe 


\section{Inhalt}

\section{Editorial}

\section{Essay}

Eva Borst

Gefährdungen des Denkens: Zum Verhältnis von Frauen und Macht

\section{Beiträge}

Rosemarie Ortner

Der Homo oeconomicus feministisch gebildet? Eine neoliberale

Herausforderung für das Subjektverständnis feministischer Bildungstheorie

Heike Kahlert

Emanzipatorisches Wissen im Schatten des Neoliberalismus:

Ökonomisierung der Kritik oder Kritik der Ökonomisierung?

Edgar Forster

Feminisierung und Geschlechterdifferenz.

\section{Work in Progress}

Stephanie Maxim

Geschlecht und Erfahrung: Zur Reifizierung von Geschlecht in der schulischen Geschlechterforschung

Angelika Paseka

Gender Mainstreaming und Transformationsprozesse im Bildungswesen:

Hoffnungen und Realitäten

Dorle Klika

Fächerwahl im Lehramtsstudium - Zementierung der

Geschlechtersegregation? 
Karin Priem

Almersia 1994-2003: Migration und biografisches Bilderwissen in

Fotografien von Rineke Dijkstra

Sylvia Bürkler

Die Genfer Frauenrechtlerinnen zu Beginn des 20. Jahrhunderts

\section{Rezensionen}

Christine Rabl

Rezension zu Mona Singer: Geteilte Wahrheit. Feministische Epistemologie,

Wissenssoziologie und Cultural Studies, Wien: Löcker 2005

Bettina Hünersdorf

Rezension zu Anja Tervooren: Im Spielraum von Geschlecht und Begehren.

Ethnographie der ausgehenden Kindheit, Weinheim: Juventa 2006

Susanne Maurer

Rezension zu Barbara Rendtorff: Erziehung und Geschlecht. Eine

Einführung, Stuttgart: Kohlhammer 2006

Karin Manz

Rezension zu Anne Schlüter (Hg.): Bildungs- und Karrierewege von

Frauen. Wissen-Erfahrung-biographisches Lernen, Opladen: Verlag

Barbara Budrich 2006

Marcus Rieger-Ladich

Rezension zu Sabine Hark: Dissidente Partizipation. Eine

Diskursgeschichte des Feminismus, Frankfurt am Main: Suhrkamp 2005 ...

Sabina Larcher

Rezension zu Marina d'Amelia: La Mamma, Bologna: Società editrice il Mulino 2005

Rebekka Horlacher

Rezension zu Yvonne Leimgruber: In pädagogischer Mission. Die Pädagogin Rosette Niederer-Kasthofer (1779-1857) und ihr Wirken für ein „frauengerechtes“ Leben in Familie und Gesellschaft, Bad Heilbrunn: Klinkhardt 2006

\section{Ankündigung der nächsten Bände}

Jahrbuch Band 4 


\title{
Feminisierung und Geschlechterdifferenz
}

\author{
Edgar Forster
}

„,Männlichkeiten“ und ,Weiblichkeiten` sind historische Geschlechtskrankheiten.“
Christina Thürmer-Rohr

„Mein Cyborgmythos handelt also von überschrittenen Grenzen, machtvollen Verschmelzungen und gefährlichen Möglichkeiten, die fortschrittliche Menschen als einen Teil notwendiger politischer Arbeit erkunden sollten.“

Donna Haraway

In einer „Grundsatzdebatte“ über den Verdacht der Feminisierung des Lehrberufs in der Neuen Zürcher Zeitung vom 20. Januar 2004 schließen Sabina Larcher und Kathrin Schafroth ihr Eingangsstatement mit dem Satz: „Denn die Bildungsfrage kann durch stereotype Rollenbilder rasch zu einer Geschlechterfrage werden“ (S. 41). Dieser Satz enthält zwei Botschaften: Die Feminisierungsdebatte transformiert die Bildungsfrage in eine Geschlechterfrage und sie produziert stereotype Rollenbilder. Eine stärkere Interpretation des Satzes besagt: Um die Bildungsfrage in eine Geschlechterfrage übersetzen zu können, muss das Geschlechterverhältnis stereotypisiert werden. Am meisten überrascht die Forderung, die Bildungsfrage nicht als Geschlechterfrage zu formulieren und damit eine feministische Forderung auf den Kopf zu stellen, der zufolge Bildungsfragen immer auch als Geschlechterfragen zu denken seien. In den Beiträgen der NZZ-Beilage wird aber vom Geschlecht und dem Geschlechterverhältnis ständig gesprochen; keine Rede davon, dass Bildungsfragen nicht auch geschlechtsspezifisch diskutiert werden sollen. Dieses Missverhältnis muss in einem Unterschied begründet sein, der darin besteht, wie Geschlecht als analytische Kategorie konzeptualisiert wird. In der Feminisierungsdebatte wird die Geschlechterordnung durch eine Trennung von Weiblichkeit und Männlichkeit, denen jeweils eindeutig unterscheidbare Attribute zugeordnet werden können, stabilisiert. Ob diese Attribute biologisch fundiert sind oder durch die Macht der Sozialisation hergestellt werden, spielt dabei nur eine Nebenrolle. Über den problematischen Zusammenhang von Feminisierungsdebatte und Geschlechterdifferenz macht 
Jürgen Oelkers (vgl. 2004) aufmerksam, wenn er in der gleichen Beilage von einer „Scheindebatte“ spricht: Es gebe keine stichhalten Belege dafür, warum zu viele Lehrerinnen für Schule und Schüler schädlich seien und was zuviel oder zuwenig konkret bedeute. Und Rita Casale (vgl. 2004) zeigt im Vergleich mit dem italienischen Bildungswesen mit dem europaweit höchsten Anteil an weiblichen Lehrkräften, dass daraus nur unter bestimmten historischen und gesellschaftlichen Bedingungen eine Diskussion über das Geschlechterverhältnis im Bildungswesen entsteht.

Um den bloßen Umstand festzuhalten, dass es einen Überhang an Lehrerinnen gibt, hat die deskriptive Kategorie ,Feminisierung ' geringe Erklärungskraft, denn das Ungleichgewicht zwischen Männern und Frauen ist für sich genommen unbestimmt. Als Prozesskategorie verbindet sich mit Feminisierung dagegen eine Veränderung von Kräfteverhältnissen zwischen den Geschlechtern, und Feminisierung kann dafür als Grund oder Effekt betrachtet werden.

Das Wort ,Feminisierung ' beschreibt verschiedene gesellschaftliche Sachverhalte und ebenso unterschiedlich wird das damit artikulierte Geschlechterverhältnis gedacht. Feminisierung der Armut: Frauen sind in stärkerem Maße von Armut betroffen als Männer in ähnlichen Situationen. Die Funktion der Kategorie Geschlecht unterscheidet sich dabei wesentlich von der Funktion der Geschlechterkategorie in der Debatte über die Feminisierung des Lehrberufs. Um systematische Diskriminierung, Ausgrenzung und Armut zu erklären, werden die Kategorien sex, race, class verknüpft (vgl. Gimenez/Hälg 2003). Frau-Sein macht in Bezug auf Armut einen Unterschied, und zwar einen einseitigen Unterschied, denn um Armut hinsichtlich Geschlecht zu erklären, hilft nicht der relationale Bezug auf Mann-Sein, sondern auf patriarchale Strukturen, die den Hintergrund dafür bilden, dass FrauSein einen Unterschied macht. Während hier nicht die relationale Kategorie gender im Vordergrund steht, sondern der einseitige Unterschied, der sich auf sex bezieht, wird der Begriff ,Feminisierung' in der Bildungsforschung und Bildungspolitik auf umgekehrte Weise zu einem politischen Kampfbegriff: Er operiert mit der Relation Weiblichkeit versus Männlichkeit und dem Zahlenverhältnis von Männern zu Frauen.

Die These meines Beitrags ist: Als analytische Kategorie bezeichnet das Wort ,Feminisierung ' die Auswirkungen eines grundlegenden Wandels von Arbeits- und Lebensverhältnissen auf die Geschlechterordnung und als politische Kategorie wird der Feminisierungsverdacht dazu benützt, die Geschlechterordnung durch eine Politik der Repräsentation der Geschlechterdifferenz zu stabilisieren. Die folgende Rekonstruktion der Feminisierungsdebatte setzt deswegen bei zwei Fragen an: Auf welche Weise werden in der 
Feminisierungsdebatte Geschlecht und Geschlechterdifferenz konzeptualisiert? Wie sind die Feminisierungsdebatte und die durch sie artikulierte Geschlechterordnung mit dem gesellschaftlichen Wandel von Arbeits- und Lebensverhältnissen verknüpft?

Um beide Fragen beantworten zu können, wird im ersten Kapitel die Feminisierungsdebatte im Bildungswesen zunächst unter folgenden Aspekten diskutiert: Was wird unter Feminisierung verstanden, welche Verhältnisse werden damit beschrieben, welche Definition von Geschlecht liegt der Debatte zugrunde und welche Schlüsse werden aus den Analysen gezogen? Diese Rekonstruktion wird zeigen, dass mit dem Feminisierungsverdacht auch eine Geschlechterlogik reproduziert wird, die asymmetrische Machtverhältnisse zwischen Männern und Frauen verteidigt. Dabei handelt es sich nicht um eine akzidentielle Begleiterscheinung der Feminisierungsthese, sondern, wie die Analyse der Lenzenschen Version von Feminisierung als Maternalisierung im zweiten Kapitel zeigt, eine wesentliche Voraussetzung für das Funktionieren des Feminisierungsdiskurses in der Pädagogik. Dieser Diskurs lässt sich durch eine charakteristische Verschiebung beschreiben: Die Ontologisierung des Geschlechterverhältnisses stützt den pädagogisch adressierten Feminisierungsverdacht und verschleiert die vielfältigen Formen der Feminisierung, die die Transformation der Arbeitsgesellschaft begleiten und Hierarchien zwischen Männern und Frauen ebenso reproduzieren wie Spaltungen innerhalb der Männer und innerhalb der Gruppe der Frauen. Im dritten Kapitel wird deswegen Feminisierung im Kontext der aktuellen ökonomischen Umwälzungen reartikuliert, um schließlich einige Konsequenzen für eine Politik der Geschlechterdemokratie zu diskutieren.

\section{Die Feminisierungsdebatte im Bildungswesen}

Die Feminisierung des Lehrberufs, des Bildungswesens, der Pädagogik und Erziehungswissenschaft ist nicht nur Gegenstand medialer Debatten, sondern auch theoretischer und empirischer Untersuchungen sowie von Expertisen und Berichten. Insbesondere vor dem Hintergrund von Ergebnissen aus international vergleichenden Schulleistungsstudien werden vor allem der Zusammenhang zwischen dem hohen Anteil von Lehrerinnen und den Schulleistungen von Jungen sowie der Zusammenhang zwischen „weiblichem“ Unterrichtsstil, Klassen- bzw. Schulklima und auffälligem Verhalten von Jungen diskutiert. Diefenbach und Klein (vgl. 2002) haben festgestellt, dass Jungen ihre Sekundarschulausbildung häufiger ohne oder mit Hauptschulabschluss 
und seltener mit einem Realschulabschluss oder mit der Hochschulreife abschließen als Mädchen. Dafür werden zwei Erklärungen angeboten: Unterschiedliche Bildungsabschlüsse von Jungen und Mädchen korrelieren mit dem Anteil männlicher Grundschullehrer, und es gibt einen positiven $\mathrm{Zu}$ sammenhang zwischen Bildungsabschluss und Arbeitslosenquote eines Bundeslandes. Hier interessiert, wie das Geschlechterverhältnis gedacht wird, das den ersten Erklärungsansatz theoretisch stützt.

Leistungsschwächen und Verhaltensprobleme von Jungen werden im Beitrag ausschließlich in Beziehung zu Mädchen formuliert, als „Nachteil“ von Jungen gegenüber Mädchen und nicht in Bezug auf Leistungsstandards, auf vorgegebene Normen und Regeln. Der Nachteil der Jungen ist der Vorteil der Mädchen, lautet die Formel für ein Geschlechterverhältnis, das in Form einer Gegensätzlichkeit von Männlichkeit und Weiblichkeit konstruiert wird. Männliche Attribute von Jungen werden negativ sanktioniert und das heißt umgekehrt, dass weibliche Attribute von Mädchen positiv wahrgenommen werden. Der Nachteil entstehe Jungen daraus, dass Schule einseitig Weiblichkeit fördere und Männlichkeit zurückweise - durch weibliche Lehrkräfte, die mit dem Jungenverhalten nicht zurechtkommen, durch einen Unterrichtsstil und ein Schulklima, die Jungen keinen Platz zur Entfaltung ihrer Geschlechtsidentität bieten. Der Nachteil von Jungen entstehe durch eine systematische Verzerrung der Leistungsbeurteilung und durch die negative Wahrnehmung von Verhaltensweisen, die den schulischen Alltag stören: ,,[...] und möglicherweise werden Lehrerinnen durch solches Verhalten stärker irritiert als Lehrer, wenn sie als Maßstab die eigene geschlechtsspezifische Sozialisation heranziehen" (ebd., S. 950). Das Problem ist nicht mehr das Verhalten von Jungen, sondern die durch weibliche Sozialisation verursachten übersensiblen Reaktionen auf Schüler. Bedeutet dies, dass Männlichkeitsattribute derart dominant sind, dass Jungen zu Außenseitern mutieren, weil sie nicht fähig sind, soziale Situationen wahrzunehmen, Spielregeln des Zusammenlebens einzuhalten und sich auf Praktiken des Aushandelns einzulassen, die eine erfolgreiche Schullaufbahn ermöglichen? Warum untergraben eine offene Diskussionskultur, die Thematisierung von Geschlechterverhältnissen in der Schule, die Zurückweisung von Aggression und Gewalt das Selbstverständnis von Jungen? Weil sie ganz anders sind? Weil sie nicht anders können? Und warum begreifen sie sich gegenüber Mädchen als zurückgesetzt? Und nicht aufgrund bestimmter Normen, die sie verletzen?

In diesem Geschlechterkonzept werden gesellschaftliche Verhältnisse ausgeblendet, indem Machtverhältnisse als Persönlichkeitseigenschaften nach innen verlagert und als scheinbar natürliche Geschlechterspannung darge- 
stellt werden. Eine einseitige Unterscheidung setzt dagegen Jungen und Mädchen zu jenem gesellschaftlichen ,Untergrund ' in Beziehung, der als patriarchalischer Kontext ihr Handeln strukturiert und durch ihr Handeln verändert wird. Die Geschichte von sich wiederholenden Differenzierungspraktiken, die männliche und weibliche Identität als aufeinander bezogene Identitäten konstituieren, lässt sich nur durch einen gemeinsamen Referenzpunkt verstehen. Wenn man den Blick auf den gemeinsamen, differenzbildenden ,Untergrund' lenkt, der durch Bildungsinstitutionen sowohl repräsentiert als auch problematisiert wird, dann ließe sich die Gleichsetzung Nachteil für Jungen = Vorteil für Mädchen aufbrechen: Könnte es nicht sein, dass sich für Mädchen mit Bildung andere Zukunftsperspektiven verbinden als für Jungen, dass Bildung für einige von ihnen mit einem Emanzipationsanspruch verbunden ist, der umgekehrt Jungen als bedrohlich erscheinen muss? Was wäre, wenn Schule tatsächlich ihre Selektions- und Platzierungsfunktion nur auf der Basis von Wissens- und Kompetenzerwerb realisieren würde? Zehrten Jungen nicht zu lange von einer Geschichte männlicher Dominanz, deren Dividende auch jenen Männern zugute kommt, die nicht an vorderster Front für ihre Vormachtstellung kämpfen? Solange Schule diese Dominanzverhältnisse reproduziert, und zwar unabhängig davon, wie erfolgreich Jungen und Mädchen die Schule absolvieren, entstehen für Jungen keine Nachteile. Könnte es also sein, dass Erfahrungen von Jungen und Mädchen mit dem Alltag einer auf Dominanz basierenden Geschlechterordnung ein ambivalentes Verhältnis zu Schule, Bildung, zu LehrerInnen und zum Wissen strukturieren, weil sich die Reproduktion herrschender Ordnungen mit ihrer Problematisierung verbindet? Was also wäre, wenn Schule von einer postgender-Welt träumen würde? Nicht von einer Welt ohne Geschlecht oder einer der geschlechtlichen Indifferenz, sondern von Differenzen, die sich nicht mehr auf ein Geschlechterverhältnis des Gegensatzes, der Ähnlichkeit oder der Analogie stützen und sich nicht mehr auf eine gegebene Identität berufen würden? Dies entzöge jeglicher Dominanz den Boden.

Die zentrale Operation in der Feminisierungsdebatte besteht nun gerade darin, die relationale Beziehung zwischen Männern und Frauen, zwischen Männlichkeit und Weiblichkeit stark zu machen. Ihren plakativen Ausdruck findet Feminisierung im Zwölften Kinder- und Jugendbericht aus Deutschland, und fundiert wird die Festschreibung eines relationalen Begriffs von Geschlecht durch die Setzung einer natürlichen Geschlechterdifferenz, die im Sozialen wiederkehrt und eine unhintergehbare Differenz der Geschlechter erzeugt, die es - das ist der überraschende Schluss - in der Erziehung zu berücksichtigen gelte: „Ein bewusst das Geschlecht differenzierendes Erzie- 
hungsverhalten erscheint geradezu notwendig, um bei beiden Geschlechtern $\mathrm{zu}$ einer gelingenden Entwicklung beizutragen" (Zwölfter Kinder- und Jugendbericht 2005, S. 163). Als Folge davon gelten den AutorInnen des Berichts feminine Stereotype von weiblichen und männlichen ErzieherInnen in Kindertageseinrichtungen als ein großes Problem (vgl. ebd.). Eva Borst (vgl. 2006, S. 50f.) hat darauf hingewiesen, dass solchermaßen theoretisierte Sozialisationsprozesse von ihrem emanzipatorischen Gehalt abgeschnitten und in natürliche Entwicklungslogiken verwandelt werden. Geschlechterentwicklung ist kein aktiver, wechselvoll verlaufender Prozess des Aushandelns widersprüchlicher, offener Identitätsformen in einem hierarchisch strukturierten Machtraum, sondern die Entfaltung des je Gegebenen. Dabei müssen die Widersprüche der Geschlechterdifferenz, ihre Fiktionalität und Funktion als Legitimation für Machtverhältnisse verleugnet und kaschiert werden. „Geschlechtsspezifische Selektionsmechanismen erscheinen dann nicht mehr als Problem gesellschaftlicher Segregation, sondern als natürliche Einflussfaktoren“ (ebd., S. 51). Erziehung ist die Entfaltung und Demonstration einer zur zweiten Natur gewordenen Programmierung gender.

KritikerInnen der Feminisierungsdebatte warnen davor, die Krise der Repräsentation des Geschlechterverhältnisses als Beschreibung der realen Geschlechterordnung zu interpretieren. In mehrfacher Hinsicht sei Zurückhaltung angebracht: Erstens bildet die im Zuge des Feminisierungsverdachtes entstandene Homogenisierung der Geschlechtergruppen die Realität nicht angemessen $a b$, denn Differenzen innerhalb der Gruppe der Jungen und der Mädchen werden ebenso kaschiert wie Gemeinsamkeiten zwischen Jungen und Mädchen. Diese Abstraktion ermöglicht erst die Repräsentation einer hierarchisierenden Relation zwischen den Geschlechtern und sie marginalisiert andere Kategorien der gesellschaftlichen Allokation von Macht. Zweitens weist Robert Connell darauf hin, dass die Vorteile aus der Patriarchatsdividende mit Kosten verbunden sind; wenn man nur auf die Kosten schaue, entstehe der Eindruck von Benachteiligung (vgl. Schneider 2002, S. 465). Drittens: Claudia Schneider (vgl. 2002) beurteilt den geschlechterdemokratischen Status quo schulischer Organisationsstruktur und -kultur skeptisch. Der hohe Frauenanteil im Lehrberuf habe die hierarchische Differenz zwischen Männern und Frauen nicht ernsthaft aufgebrochen, vielmehr seien nur die „Geschlechterreviere“ (Horstkemper) neu abgesteckt worden. Viertens: Auch die Einschätzung der Situation von underachieving boys (vgl. Reed 2006) ist nicht so eindeutig, wie sie bei Diefenbach und Klein dargestellt werden. Insbesondere sei es Mädchen bislang nicht gelungen, ihr besseres Abschneiden in berufliche Karrieren umzusetzen. Nach wie vor stoßen sie an eine gläserne 
Decke, sie arbeiten öfters in prekären Arbeitsverhältnissen und an der Einkommensverteilung zwischen Männern und Frauen hat sich nur wenig geändert. Schließlich zeigen Interpretationen des sogenannten ,Jungenproblems und der ,Krise ' von Männlichkeit (Männer werden öfters kriminell als Frauen, Jungen gelten im Unterschied zu Mädchen häufiger als verhaltensauffällig usw.) die Konfusion der Feminisierungsdebatte: Sind dies durch einen weiblichen Blick gebrochene inadäquate Zuschreibungen? Handelt es sich um eine Identitätskrise von Männern, ausgelöst durch Frauen, die die Geschlechterordnung verändern wollen? Oder wird dieses männliche Verhalten in einer sich verändernden Gesellschaft dysfunktional? Sie lassen sich auch als Versuch einer „männlichen Resouveränisierung“ (Forster 2006) deuten, als ein letztes Hegemoniestreben mit untauglichen Mitteln. Schließlich: Ist nicht die Darstellung von problematischen männlichen Verhaltensweisen als Geschlechterfrage dann fragwürdig, wenn sich daran sofort die Frage nach der Verantwortung von Frauen knüpft?

Trotz der Unterschiede folgen die Interpretationen ähnlichen Argumentationsmustern: Unbestritten hat der Faktor Gesellschaft eine neue Macht erlangt. Genderism durchzieht noch die letzte Faser schulischer Interaktion (vgl. Faulstich-Wieland/Güting/Ebsen 2001), sie affiziert hinterrücks den Unterrichtsstil und die Leistungsbeurteilungen von Lehrerinnen (und Lehrern?). Gender ist zur zweiten Natur geworden, hartnäckig wie sex. Und auch wenn wir wissen, dass sex und gender zwei voneinander getrennte Kategorien sind, bilden sie durch einen langen Prozess der Naturalisierung ein unauflösbares Paar, das schließlich im Konzept des Habitus seinen theoretischen Niederschlag gefunden hat. Nicht mehr die Macht der Natur, sondern die Macht des Sozialen regelt die Geschlechterordnung und die theoretisch gewonnene Einsicht in die Veränderbarkeit der Welt durch die Zurückweisung der Macht des biologischen Geschlechts wird mit der „Wiederkehr der Natur im Sozialen" (Casale/Forster 2006) bezahlt.

Die Feminisierungsdebatte hat auf dem Boden der Gender Studies und der von den Medien ausgerufenen Krise der Männer dazu beigetragen, die Geschlechterfrage in der Pädagogik auch bei denjenigen salonfähig zu machen, die dem Feminismus distanziert gegenüber stehen - wenn auch auf andere Weise, als sich feministische und männlichkeitskritische ForscherInnen und PraktikerInnen erhofft hatten, denn Bildungsfragen als Geschlechterfragen zu reformulieren, kann auch bedeuten, jene cyborgphobe Geschlechterlogik zu reproduzieren, die Hierarchien zwischen Männern und Frauen befestigt. Es ist also notwendig, die der Feminisierungsdebatte zugrunde liegende Theorie systematisch darauf hin zu untersuchen, wie sie Geschlechterlogiken produziert. 


\section{Die Intrusion der Frauen}

In der Erziehungswissenschaft formuliert Dieter Lenzen (vgl. 2000) die theoretisch weitreichendste Hypothese zur Feminisierung (als Maternalisierung), die für das Verständnis der Pädagogik im 20. Jahrhundert von zentraler Bedeutung sei. Aus einer Reihe von Gründen habe sich die Repräsentanz von Müttern im 20. Jahrhundert erheblich erweitert und die Gesellschaften strukturell verändert, denn die maternal bestimmte familiäre Logik der totalen Kommunikation - alles, was gesagt wird, ist wichtig - und der damit verbundenen Geheimnislosigkeit habe mit dem Eindringen von Frauen in andere gesellschaftliche Teilsysteme die dort wirkenden Codes dermaßen verändert, dass nichts Persönliches exkommuniziert werden dürfe. Der Wohlfahrtsstaat sei dafür ebenso ein Indiz wie die Gesundheitsfürsorge, die Humanwissenschaften oder das Erziehungssystem. Immer mehr breite sich die Logik des Familialen aus, die allerdings mit einer Paradoxie behaftet sei, dem unerfüllbaren Wunsch nach Unabhängigkeit. Im Begriff der Mündigkeit, die es nur als Einsicht in Unmündigkeit gebe, habe die Erziehungswissenschaft diesem Paradox eine theoretische Fassung gegeben.

Die Rekonstruktion konzentriert sich auf die Artikulation von Geschlecht und Geschlechterdifferenz. Andere Aspekte bleiben im Hintergrund, etwa die theoretischen Implikationen, die durch den Umstand gegeben sind, dass Lenzen die Feminisierungsdebatte systemtheoretisch rekonstruiert. Meine Lesart artikuliert die These, dass die Argumentationslogik darauf beruht, dass erstens das Verhältnis von sex und gender unaufgelöst und als Spannungsmoment bestehen bleibt; dass zweitens eine zügellose Differenz durch eine Repräsentation gebändigt wird und Feminisierung den Knotenpunkt dieser Politik der Repräsentation bildet; und dass drittens Dominanzrelationen und damit der Kampf zwischen den Geschlechtern unterdrückt werden. Die folgende Argumentation wird durch einen Begriff von Differenz bestimmt, der nicht eine bestimmte Einheit oder ein Ganzes voraussetzt, die die differenten Elemente definieren. Deswegen wird die relationale Bestimmung der Geschlechter bereits als Effekt einer bestimmten Repräsentationspolitik gesehen, die dazu neigt, gender als Ensemble von Attributen zu sehen, die Individuen auszeichnen. Der Feminisierungsverdacht im Bildungswesen operiert aber genau mit dieser Repräsentationslogik und sie soll deswegen einer grundsätzlichen Kritik unterzogen werden.

Die Feminisierungsthese von Lenzen ist deswegen von Interesse, weil sie von einem kruden Biologismus weit entfernt ist. Aber weil an jeder Stelle des Textes viel Sorgfalt darauf verwendet wird, Mütterlichkeit und Weiblichkeit 
nicht mit Frauen kurzzuschließen, fällt die Spannung zwischen Frauen und Maternalität sofort auf. Feminisierung löst sich von Frauen, um wieder bei ihnen zu landen. Man könnte dies die heimliche „Intrusion“ der Frauen bzw. Mütter nennen, die das systemtheoretische Feminisierungsmodell stützen, antreiben und aufrecht erhalten und eine biologische Geschlechterdifferenz reproduzieren, die dann hinter der Kommunikation der Teilsysteme verschwindet.

Der Begriff „Intrusion“ ist dem Text entnommen: ,[...] können wir prüfen, ob zeitgleich mit der Intrusion von Frauen in bestimmte soziale Teilsysteme Kommunikationen der geschilderten Art stattfinden und ob sich damit das Kommunikationsmedium jeweils geändert hat" (Lenzen 2000, S. 129; Hervorhebung: E.F.). Dabei markiert das Adverb „zeitgleich“ eine Vorsichtsmaßnahme gegenüber Kausalisierung. Und trotzdem werden zwei Sachverhalte dadurch miteinander in Beziehung gesetzt, so dass eins das andere affiziert. Intrusion bezeichnet dieses Affizieren als gewaltsamen Akt des widerrechtlichen Eindringens in einen fremden Bereich.

Die Argumentationslogik entkommt den Widersprüchen des sex-genderKonzepts nicht, denn gender funktioniert als Konzept nur, wenn es durch sex gestützt wird. Weiblichkeit ist ohne Frauen nicht zu haben und Mütterlichkeit nicht ohne Mütter und Mutterschaft, weil gender nur als Attribut gedacht wird, als eine Reihe von Eigenschaften, die Frauen oder Männern zukommen. Judith Butlers Dekonstruktion des sex-gender-Verhältnisses müsste dort fruchtbar gemacht werden, wo sich das Attribut gender in ein Prädikat verwandelt. Während das Attribut eine Qualität ausdrückt und auf ein Wesen verweist, ist das Prädikat ein Ereignis, und Ereignisse sind Verhältnisse zur Existenz und Zeit. Das Prädikat ist eine Tat, eine Bewegung, eine Veränderung und nicht der Zustand des Bewegens. Es ist ein Verb, die Aussage selbst, und man kann sagen, dass die Welt die Prädikation ist, während die besonderen Prädikate die Weisen der Welt sind. Das Subjekt verläuft von einem Prädikat zum anderen, und das Prädikat löst als Grund in jedem Subjekt das Attribut als dessen Wesen ab (vgl. Deleuze 1995, S. 88ff.). Der Unterschied zwischen Attribut und Prädikat ist der Unterschied zwischen gender und doing gender, aber die konkreten Praktiken stellen nicht Männlichkeiten oder Weiblichkeiten her, sie machen nicht gender als Attribut begreiflich, denn zwischen gender und doing gender gibt es eine ebenso tiefe Kluft wie zwischen Attribut und Prädikat. Doing gender sind Praktiken, die Frauen und Männer in Machtverhältnissen situieren, während gender eine Semantik darstellt, die die Bewegungen des doing gender überformt, verschleiert, legitimiert oder delegitimiert, indem sie die Praktiken des doing gender als Reprä- 
sentation einer Geschlechtsidentität begreift, währenddessen die Praktiken des doing gender jeder Identität vorausgehen und, weil es sich um Praktiken der Wiederholung handelt, jede Form der Repräsentation unterminieren. Doing gender sind Praktiken, die gesellschaftliche Ordnungen produzieren: Machtverhältnisse, Hierarchien, Distanz, Abstand, Nähe, Anziehung, AbstoBung. Sie beziehen sich nicht auf Weiblichkeit und Männlichkeit, sondern auf sex, auf Frau und Mann, aber nicht derart, dass doing gender in der Lage wäre, das biologische Geschlecht auszudrücken, doing gender ist nicht das Signifikat des Signifikanten sex und es bringt auch nicht sex als Effekt hervor. Noch vor jeder Identifizierung mit einer Geschlechterlogik produzieren Praktiken des doing gender Intensitäten, die durch Differenzen gebildet werden, die selbst auf andere Differenzen verweisen (vgl. Deleuze 1992, S. 156). Es gibt keine erste Differenz, so dass man nicht sagen kann, doing gender verweise auf den Körper - biologische Differenzen erzeugen selbst Intensitäten -, aber sie verknüpfen sich mit anderen Differenzen. Bevor sich Geschlecht in eine Politik der Geschlechterrepräsentation und der Heteronormativität einfangen lässt, muss man Geschlecht als Differenz im Sinne der Bestimmung als einseitiger Unterscheidung fassen. Nur in diesem Sinn sind der Körper und seine Grenzen zentral. Doing gender und schließlich gender sind auf unterschiedliche Weisen Politiken der Repräsentation von Geschlechtsidentität: doing gender ist das unterminierende Element der Repräsentation, während gender das fixierende, attribuierende Element einer ,sozialen Schließung“ bildet (Weber, M. 1972, S. 201; vgl. auch Weber, C. 1987). Der Körper, das biologische und das soziale Geschlecht sind Abstraktionen, die man aufbrechen müsste, um dahinter Gefüge sichtbar zu machen, die Praktiken, Gefühle, Begehren als Verhältnisse organisieren und funktionieren lassen.

Die erste Operation im Text besteht darin, diese Gefüge durch einen Abstraktionsprozess unkenntlich zu machen. Auf diese Weise werden Maternalität und Feminisierung zu Attributen eines Systems, die von den handelnden Personen vollzogen werden. Der Grund für die Maternalität bleibt ebenso dunkel wie die Weisen, in denen sie sich äußert, jeglichen Bereich erfassen und sich endlos fortpflanzen. Die Macht des Maternalen steigt ins Unermessliche, um schließlich eine hundertjährige Geschichte und auch noch das „Führerprinzip“ zu erklären (vgl. Lenzen 2000, S. 141).

Was im Feminisierungsmodell ausgeschlossen werden soll, dringt unaufhörlich ein: die zügellose Differenz, die durch keine Repräsentation gebändigt werden kann. Darauf reagiert die zweite Operation: mit einer Politik der Repräsentation. Die größte Gefahr droht von der Entdifferenzierung gesell- 
schaftlicher Teilsysteme und der Entgrenzung eines Teilsystems, das alle anderen Systeme infiziert und deren binären Codes zerstreut. Das Maternale strukturiere familiale Kommunikation auf besondere Weise: Sie mache die Person nicht als Funktion, sondern per se zum Thema. Deswegen können alle Handlungen und alles Persönliche zum Gegenstand der Kommunikation werden und schließlich unterliege alles der Beobachtung zweiter Ordnung. Wenn die maternal strukturierten Kommunikationsregeln andere Teilsysteme affizieren, dann zerstreuen sie deren Kommunikationscodes: die Binarität von heilbar/unheilbar im Gesundheitswesen durch die Stärkung prophylaktischer Arbeit, im politischen System das Schema Amtsinhaber/Unterworfene bzw. Überlegene/Unterlegene durch den modernen Wohlfahrtsstaat, das Wissenschaftssystem, deren Codes wie Faktizität, Geltung und Wahrheit durch Meinungen, Befindlichkeiten und Wertungen affiziert werden. Und schließlich sei die Pädagogik ein Feld par excellence für die Dominanz des Maternalen. Mit der Inthronisation des „Auges der großen Mutter“, deren Umarmung sich niemand entziehen könne, wird eine alles umfassende Teilung der Gesellschaft nach Geschlechtern entworfen.

Die Politik der Repräsentation bändigt das Wuchern der Differenzen, indem sie Binarität, Grenzen, Unterscheidungen, die sich auf eine gegebene Identität berufen, herstellt. Sie kanalisiert und ritualisiert Kämpfe zwischen den Geschlechtern, um die gefährlicheren Kämpfe zu unterdrücken, die sich gegen die Repräsentation selbst wenden. Problematisch ist die Feminisierung nicht deswegen, weil sie Codes umkehrt, sondern weil sie sie vervielfältigt, ihren Geltungsbereich ausdehnt und ihre Ränder ausfranst. Auf diese Weise implodieren die gesellschaftlichen Teilsysteme, neue Recodierungen entstehen und sie sind anderen Souveränitätsmaschinen unterworfen.

Das bedeutet, dass die Debatte über die Feminisierung der Pädagogik im Kontext der aktuellen gesellschaftlichen Transformationsprozesse diskutiert werden muss, um beurteilen zu können, ob die Krise der Politik der Repräsentation des Geschlechterverhältnisses, auf die die Feminisierungsdebatte mit misogynen, aber zahnlosen Waffen reagiert, nicht als Effekt einer grundlegenden Umgestaltung gesellschaftlicher Verhältnisse begriffen werden muss.

\section{Feminisierung und affektive Arbeit}

Feminisierung wird im Zusammenhang mit einem grundlegenden Wandel der Arbeit diskutiert, der sich entlang zweier Achsen vollzieht, die bislang cha- 
rakteristisch für die Frauenarbeit waren: Immaterielle Arbeit nimmt an Bedeutung zu und es gibt neue Formen der Ausbeutung und Unterwerfung der Arbeitskraft von Frauen, die immer schon mit Prekarisierung und Flexibilisierung verknüpft war (vgl. Sauer 2006, Haug 2003). Immaterielle Arbeit setzt sich aus drei Formen zusammen: Die Informatisierung der industriellen Produktion, Tätigkeiten mit analytischen und symbolischen Anforderungen, die zum einen eine kreative und intelligente Handhabung verlangen, zum anderen Routinetätigkeiten sind, und schließlich die Produktion und Handhabung von Affekten. Affektive Arbeit produziert über Kommunikation und Interaktion den „sozialen Kitt“ einer Gesellschaft (vgl. z.B. Hardt 2004, S. 184f.). Durch immaterielle Arbeit löst sich die traditionelle Trennung von Ökonomie und Kultur auf, Arbeitszeit und Lebenszeit überlagern sich, so dass von Arbeitszeit im strengen Sinn nicht mehr gesprochen werden kann. Die Folgen sind u.a. Mehrfachbelastungen vor allem für Frauen und die Etablierung von grauen Arbeitsmärkten mit niedrigen oder unbezahlten Tätigkeiten in der Hausarbeitsökonomie, obwohl affektive Arbeit zu einem wichtigen Faktor in der Produktion geworden ist.

Wenn man den Wandel von Arbeits- und Lebensbedingungen auch als Feminisierung begreift, so nicht deswegen, weil der Anteil weiblicher Arbeitskräfte auf dem Arbeitsmarkt stark ansteigt, sondern weil ,die Dispositive der Unterwerfung, die in historischer Perspektive vor allem Frauen betreffen, ausgeweitet werden“ (Revel 2004, S. 256). Das heißt, dass Gefühle, Tätigkeiten und Beziehungen, die zuvor radikal von ,produktiver' Arbeit getrennt waren, nun in die Produktionssphäre übergehen. Als Folge davon implodiert die durch die getrennten Sphären produzierte Geschlechterteilung. Das betrifft den Unterschied zwischen produktiver und reproduktiver Arbeit, zwischen Maschinenarbeit und Arbeit mit Menschen, zwischen kognitiver und affektiver Arbeit, zwischen Arbeit und Nicht-Arbeit, zwischen Familie und Arbeit, zwischen Familienernährer-Arbeit und Zuarbeit, zwischen privater und öffentlicher Sphäre, zwischen ,privatem“ und „öffentlichem“ Patriarchat (Fraser 1994, S. 223).

Das Modell der Intrusion des Familialen in alle Gesellschaftsbereiche und die Zerstörung binärer Codes findet sich auch in der Analyse der ,neuen Weltordnung“ bei Hardt und Negri (vgl. 2002). Während aber Lenzen gegen die Entdifferenzierung, die er etwa im Schulsystem ortet (vgl. Vereinigung der Bayerischen Wirtschaft 2003), auf eine Politik der Repräsentation wohlgeordneter Differenzen und auf eine starke Figur des Maternalen setzt, artikuliert sich durch seine These hindurch das Gegenteil: die Zerstreuung binärer Codes. Hardt und Negri sehen darin das Ungeheuerliche eines Kampfes am Werk. Bei allen Unterschieden gibt es eine gemeinsame Botschaft: Die 
gesellschaftlichen Umwälzungen der Arbeits- und Lebenswelt unterminieren die Politik der Repräsentation des Geschlechterverhältnisses und damit männliche Hegemonie, weil die Institutionen, die für eine stabile Geschlechterordnung gesorgt haben, implodieren.

Was daraus für die Geschlechterpolitik folgt, ist offen. Aber an einigen Indizien lassen sich künftige Kämpfe erahnen: Die Feminisierungsdebatte in der Pädagogik zeigt, dass Bildung als zentrales Element des Sich-beständigselbst-neu-entwerfen-und-erfinden-Müssens wichtiger werden wird. Sie schließt vor allem jene schulischen Kompetenzen ein, bei denen Mädchen heute gegenüber Jungen punkten: Beharrungsvermögen, Fleiß, Anpassungsfähigkeit und Flexibilität. Zweitens: Männliche Resouveränisierungen nehmen zu, aber ihre Strategien und Taktiken werden sich verändern. Zu neuen Souveränitätsformen passen Versuche, auf der Ebene der Geschlechtersemantik für eine liberale Politik zu plädieren, Geschlechtercodes zu vervielfältigen, mit Anspielungen und Durchkreuzungen tradierter Codes zu spielen, um die Unhaltbarkeit dualistischer, heternormativer Geschlechterkonzepte zu demonstrieren, während gleichzeitig soziale und ökonomische Strukturen jene sozialen Institutionen stärken, die neue Geschlechterteilungen produzieren. Wenn diese neuen Formen der Segregation mit der Wucherung von Semantiken und mit individualisierenden Selbstentwürfen verknüpft werden, dann bedeutet dies die Vervielfältigung von gesellschaftlichen Spaltungen und die Zerstörung einer Öffentlichkeit, sofern diese als wirksame politische Kontrolle verstanden wird. Die Vervielfältigung von Spaltungen ist auch deshalb wirksam, weil die Rückkehr zur Identitätspolitik mit ihren alten Herrschaftsmustern keinen Ausweg bietet, wie die Feminisierungsdebatte in der Pädagogik zeigt. Drittens: Es lassen sich erste Anzeichen einer Repolitisierung erkennen. Diese wird eine Politik gegen die Repräsentation und damit gegen die Wucherung der Spaltung sein. Ihr Grundsatz: Fission Impossible.

\section{Literatur}

Borst, Eva (2006): Die vermessene Bildung, in: Badawia, Tarek/Luckas, Helga/Müller, Heinz (Hg.): Das Soziale gestalten. Über Mögliches und Unmögliches der Sozialpädagogik. Wiesbaden, S. 41-54

Casale, Rita (2004): „Altera Mater“ oder der Fall Italien. Bildungsarbeit als Teil der Mutterrolle, in: Neue Zürcher Zeitung, Nr. 15, 20. Januar 2004, S. 49

Casale, Rita/Forster, Edgar (2006): Der neue Mann oder die Wiederkehr der Natur im Sozialen, in: Feministische Studien - Zeitschrift für interdisziplinäre Frauen- und Geschlechterforschung, 24. Jg., H. 2, S. 185-192 
Deleuze, Gilles (1995): Die Falte. Leibniz und der Barock, Frankfurt/M.

Deleuze, Gilles (1992): Differenz und Wiederholung, München

Diefenbach, Heike/Klein, Andreas (2002): „Bringing Boys Back In“. Soziale Ungleichheit zwischen den Geschlechtern im Bildungssystem zuungunsten von Jungen am Beispiel der Sekundarschulabschlüsse, in: Zeitschrift für Pädagogik, Jg. 48, H. 6, S. 938-958

Faulstich-Wieland, Hannelore/Güting, Damaris/Ebsen, Silke: Einblicke in „Genderism“ im schulischen Verhalten, in: Zeitschrift für Pädagogik, Jg. 47, H. 1, S. 67-79

Forster, Edgar (2006): Männliche Resouveränisierungen, in: Feministische Studien Zeitschrift für interdisziplinäre Frauen- und Geschlechterforschung, 24. Jg., H. 2, S. 193-207

Fraser, Nancy (1994): Widerspenstige Praktiken. Macht, Diskurs, Geschlecht, Frankfurt/M.

Gimenez, Martha E./Hälg, Anja (2003): Feminisierung der Armut, in: Historischkritisches Wörterbuch des Feminismus, hgg. im Auftrag des Instituts für Kritische Theorie von Frigga Haug, Band 1, Hamburg, Sp. 143-156

Haraway, Donna (1995, ursprüngl. 1985): Ein Manifest für Cyborgs. Feminismus im Streit mit den Technowissenschaften, in: Dies.: Die Neuerfindung der Natur. Primaten, Cyborgs und Frauen, Frankfurt/M., S. 33-72

Hardt, Michael (2004): Affektive Arbeit, in: Atzert, Thomas/Müller, Jost (Hg.): Immaterielle Arbeit und imperiale Souveränität. Analysen und Diskussionen zu Empire, Münster, S. 175-188

Hardt, Michael/Negri, Antonio (2002): Empire. Die neue Weltordnung, Frankfurt/M.

Haug, Frigga (2003): Feminisierung der Arbeit, in: Historisch-kritisches Wörterbuch des Feminismus, hgg. im Auftrag des Instituts für Kritische Theorie von Frigga Haug, Band 1, Hamburg, Sp. 128-142

Larcher, Sabina/Schafroth, Kathrin (2004): Die Bildungsfrage - auch eine Geschlechterfrage, in: Neue Zürcher Zeitung, Nr. 15, 20. Januar 2004, S. 41

Lenzen, Dieter (2000): Das Jahrhundert der Mütter - Zur Feminisierung der Pädagogik im zwanzigsten Jahrhundert, in: Baader, Meike Sophia/Jacobi, Juliane/ Andresen, Sabine (Hg.): Ellen Keys reformpädgogische Vision. „Das Jahrhundert des Kindes" und seine Wirkung, Weinheim/Basel, S. 125-147

Oelkers, Jürgen (2004): Pythagoras in weiblicher Hand. Den Fokus auf Schüler statt Lehrpersonen legen, in: Neue Zürcher Zeitung, Nr. 15, 20. Januar 2004, S. 43

Reed, Lynn R. (2006): Troubling boys and disturbing discourses on masculinity and schooling: a feminist exploration of current debates and interventions concerning boys in school, in: Arnot, Madeleine/Mac an Ghaill, Mairtin (eds.): The RoutledgeFalmer Reader in Gender and Education, London, S. 33-48

Revel, Judith (2004): Devenir-femme der Politik, in: Atzert, Thomas/Müller, Jost (Hg.): Immaterielle Arbeit und imperiale Souveränität. Analysen und Diskussionen zu Empire, Münster, S. 255-262

Sauer, Birgit (2006): „Feminisierung“ eines männlichen Projekts? Sozialstaat im Zeitalter der Globalisierung, in: Appelt, Erna/Weiss, Alexandra (Hg.): Globa- 
lisierung und der Angriff auf die europäischen Wohlfahrtsstaaten, Hamburg, S. $67-83$

Schneider, Claudia (2002): Die Schule ist männlich?! Wiener Schulen auf dem Weg von der geschlechtssensiblen Pädagogik zur geschlechtssensiblen Schulentwicklung, in: SWS-Rundschau, 42. Jg., H. 4, S. 464-488

Thürmer-Rohr, Christina (1987): Feminisierung der Gesellschaft - Weiblichkeit als Putz- und Entseuchungsmittel, in: Dies.: Vagabundinnen. Feministische Essays, Berlin, S. 106-121

Vereinigung der Bayerischen Wirtschaft (Hg.) (2003): Bildung neu denken! Das Zukunftsprojekt, Gesamtredaktion: Dieter Lenzen, Opladen

Weber, Claudia (1987): Frauen in Männerberufen. „Soziale Schließung“ und Feminisierung, in: Hauser, Kornelia (Hg.): Viele Orte. Überall? Feminismus in Bewegung. Festschrift für Frigga Haug, Hamburg, S. 203-217

Weber, Max (1972, ursprünglich 1921): Wirtschaft und Gesellschaft. Grundriss der verstehenden Soziologie, hgg. von Johannes Winckelmann, Studienausgabe, Tübingen (5. Auflage)

Zwölfter Kinder- und Jugendbericht (2005): Bericht über die Lebenssituation junger Menschen und die Leistungen der Kinder- und Jugendhilfe in Deutschland. Hgg. vom Bundesministerium für Familie, Senioren, Frauen und Jugend 